\title{
Learning Feature Engineering for Classification
}

\author{
Fatemeh Nargesian $^{1}$, Horst Samulowitz ${ }^{2}$, Udayan Khurana ${ }^{2}$ \\ Elias B. Khalil ${ }^{3}$, Deepak Turaga ${ }^{2}$ \\ ${ }^{1}$ University of Toronto, ${ }^{2}$ IBM Research, ${ }^{3}$ Georgia Institute of Technology \\ fnargesian@cs.toronto.edu, \{samulowitz, ukhurana\}@us.ibm.com, \\ lyes@gatech.edu,turaga@us.ibm.com
}

\begin{abstract}
Feature engineering is the task of improving predictive modelling performance on a dataset by transforming its feature space. Existing approaches to automate this process rely on either transformed feature space exploration through evaluation-guided search, or explicit expansion of datasets with all transformed features followed by feature selection. Such approaches incur high computational costs in runtime and/or memory. We present a novel technique, called Learning Feature Engineering (LFE), for automating feature engineering in classification tasks. LFE is based on learning the effectiveness of applying a transformation (e.g., arithmetic or aggregate operators) on numerical features, from past feature engineering experiences. Given a new dataset, LFE recommends a set of useful transformations to be applied on features without relying on model evaluation or explicit feature expansion and selection. Using a collection of datasets, we train a set of neural networks, which aim at predicting the transformation that impacts classification performance positively. Our empirical results show that LFE outperforms other feature engineering approaches for an overwhelming majority (89\%) of the datasets from various sources while incurring a substantially lower computational cost.
\end{abstract}

\section{Introduction}

Feature engineering is a central task in data preparation for machine learning. It is the practice of constructing suitable features from given features that lead to improved predictive performance. Feature engineering involves the application of transformation functions such as arithmetic and aggregate operators on given features to generate new ones. Transformations help scale a feature or convert a non-linear relation between a feature and a target class into a linear relation, which is easier to learn.

Feature engineering is usually conducted by a data scientist relying on her domain expertise and iterative trial and error and model evaluation. To perform automated feature engineering, some existing approaches adopt guided- search in feature space using heuristic feature quality measures (such as information gain) and other surrogate measures of performance [Markovitch and Rosenstein, 2002; Fan et al., 2010]. Others perform greedy feature construction and selection based on model evaluation [Dor and Reich, 2012; Khurana et al., 2016]. Kanter et al. proposed the Data Science Machine (DSM) which considers feature engineering problem as feature selection on the space of novel features. DSM relies on exhaustively enumerating all possible features that can be constructed from a dataset, given sequences generated from a set of transformations, then performing feature selection on the augmented dataset [Kanter and Veeramachaneni, 2015]. Evaluation-based and exhaustive feature enumeration and selection approaches result in high time and memory cost and may lead to overfitting due to brute-force generation of features. Moreover, although deep neural networks (DNN) allow for useful meta-features to be learned automatically [Bengio et al., 2013], the learned features are not always interpretable and DNNs are not effective learners in various application domains.

In this paper, we propose LFE (Learning Feature Engineering), a novel meta-learning approach to automatically perform interpretable feature engineering for classification, based on learning from past feature engineering experiences. By generalizing the impact of different transformations on the performance of a large number of datasets, LFE learns useful patterns between features, transforms and target that improve learning accuracy. We show that generalizing such patterns across thousands of features from hundreds of datasets can be used to successfully predict suitable transformations for features in new datasets without actually applying the transformations, performing model building and validation tasks, that are time consuming. LFE takes as input a dataset and recommends a set of paradigms for constructing new useful features. Each paradigm consists of a transformation and an ordered list of features on which the transformation is suitable.

At the core of LFE, there is a set of Multi-Layer Perceptron (MLP) classifiers, each corresponding to a transformation. Given a set of features and class labels, the classifier predicts whether the transformation can derive a more useful feature than the input features. LFE considers the notion of feature and class relevance in the context of a transformation as the measure of the usefulness of a pattern of feature value 
and class label distributions, and transformation.

Different datasets contain different feature sizes and different value ranges. One key challenge in generalizing across different datasets is to convert feature values and their class labels to a fixed size feature vector representation that can be fed into LFE classifiers. To characterize datasets, handcrafted meta-features, fixed-size stratified sampling, neural networks and hashing methods have been used for different tasks [Michie et al., 1994; Kalousis, 2002; Feurer et al., 2015; Weinberger et al., 2009]. However, these representations do not directly capture the correlation between feature values and class labels. To capture such correlations, LFE constructs a stack of fixed-size representations of feature values per target class. We use Quantile Data Sketch to represent feature values of each class. Quantile has been used as a fixed-size space representation and achieves reasonably accurate approximation to the distribution function induced by the data being sketched [Greenwald and Khanna, 2001].

LFE presents a computationally efficient and effective alternative to other automated feature engineering approaches by recommending suitable transformations for features in a dataset. To showcase the capabilities of LFE, we trained LFE on $85 \mathrm{~K}$ features, extracted from 900 datasets, for 10 unary transformations and $122 \mathrm{~K}$ feature pairs for 4 binary transformations, for two models: Random Forest and Logistic Regression. The transformations are listed in Table 1. We empirically compare LFE with a suite of feature engineering approaches proposed in the literature or applied in practice (such as the Data Science Machine, evaluation-based, random selection of transformations and always applying the most popular transformation in the training data) on a subset of 50 datasets from UCI repository [Lichman, 2013], OpenML [Vanschoren et al., 2014] and other sources. Our experiments show that, of the datasets that demonstrated any improvement through feature engineering, LFE was the most effective in $89 \%$ of the cases. As shown in Figure 2, similar results were observed for the LFE trained with Logistic Regression. Moreover, LFE runs in significantly lesser time compared to the other approaches. This also enables interactions with a practitioner since it recommends transformations on features in a short amount of time.

\section{Related Work}

The FICUS algorithm [Markovitch and Rosenstein, 2002] takes as input a dataset and a set of transformations, and performs a beam search over the space of possible features. FICUS's search for better features is guided by heuristic measures based on information gain in a decision tree, and other surrogate measures of performance. The more recent FCTree [Fan et al., 2010] also uses a decision tree to partition the data using original or constructed features as splitting points. The FEADIS algorithm of [Dor and Reich, 2012] relies on a combination of random feature generation and feature selection. FEADIS adds constructed features greedily, and as such requires many expensive performance evaluations. The Deep Feature Synthesis component of Data Science Machine (DSM) [Kanter and Veeramachaneni, 2015] relies on exhaustively enumerating all possible new features, then performing feature selection over the augmented dataset. Cognito [Khurana et al., 2016] recommends a series of transformations based on a greedy, hierarchical heuristic search. Cognito and DSM focus on sequences of feature transformations, which is outside the scope of this paper. In contrast to these approaches, we do not require expensive classifier evaluations to measure the impact of a transformation. ExploreKit [Katz et al., 2016] also generates all possible candidate features, but uses a learned ranking function to sort them. ExploreKit requires generating all possible candidate features when engineering features for a new (test) dataset and as such, results reported for ExploreKit are with a time limit of three days per dataset. In contrast, LFE can generate effective features within seconds, on average.

Several machine learning methods perform feature extraction or learning indirectly. While they do not explicitly work on input features and transformations, they generate new features as means to solving another problem [Storcheus et al., 2015]. Methods of that type include dimensionality reduction, kernel methods and deep learning. Kernel algorithms such as SVM [Shawe-Taylor and Cristianini, 2004] can be seen as embedded methods, where the learning and the (implicit) feature generation are performed jointly. This is in contrast to our setting, where feature engineering is a preprocessing step.

Deep neural networks learn useful features automatically [Bengio et al., 2013] and have shown remarkable successes on video, image and speech data. However, in some domains feature engineering is still required. Moreover, features derived by neural networks are often not interpretable which is an important factor in certain application domains such as healthcare [Che et al., 2015].

\section{Automated Feature Engineering Problem}

Consider a dataset, $\mathcal{D}$, with features, $\mathcal{F}=\left\{f_{1}, \ldots, f_{n}\right\}$, and a target class, $\kappa$, a set of transformations, $\mathcal{T}=\left\{T_{1}, \ldots, T_{m}\right\}$, and a classification task, $\mathcal{L}$. The feature engineering problem is to find $q$ best paradigms for constructing new features such that appending the new features to $\mathcal{D}$ maximizes the accuracy of $\mathcal{L}$. Each paradigm consists of a candidate transformation $T_{c} \in \mathcal{T}$ of arity $r$, an ordered list of features $\left[f_{i}, \ldots, f_{i+r-1}\right]$ and a usefulness score.

For a dataset with $n$ features and with $u$ unary transformations, $O(u \times n)$ new features can be constructed. With $b$ binary transformations, there are $O\left(b \times P_{2}^{n}\right)$ new possible features, where $P_{2}^{n}$ is the 2-permutation of $n$ features. Given a fixed set of transformations, the number of new features and their combinations to explore, for an exact solution, grows exponentially. Hence, we make the case that a mere enumeration and trial by model training and testing is not a computationally practical option, and a scalable solution to the problem must avoid this computational bottleneck. LFE reduces the complexity of feature space exploration by providing an efficient approach for finding a particularly "good" transformation for a given set of features. Therefore, given $n$ features, for unary and binary transformations, LFE performs, respectively, $O(n)$ and $O\left(P_{2}^{n}\right)$ transformation predictions.

In order to assess the relative impact of adding new features 
across different techniques, we add as many new features as those originally in the data. For unary transformations, LFE predicts the most suitable transformation for each feature. For binary and higher arity transformations, LFE considers a random sample of all combinations of features, finds the paradigm for each combination and selects top- $k$ useful ones. In the following section, we describe how LFE learns and predicts useful transformations for features.

\section{Transformation Recommendation}

LFE models the problem of predicting a useful $r$-ary transformation $T_{c} \in \mathcal{T}_{r},\left(\mathcal{T}_{r}\right.$ is the set of $r$-ary transformations in $\left.\mathcal{T}\right)$, for a given list of features $\left[f_{1}, \ldots, f_{r}\right]$ as a multi-class classification problem, where the input is a representation of features, $R_{\left[f_{1}, \ldots, f_{r}\right]}$, and output classes are transformations in $\mathcal{T}_{r}$. LFE takes a one-vs-rest approach [Rifkin and Klautau, 2004]. Each transformation is modelled as a Multi-Layer Perceptron (MLP) binary classifier with real-valued confidence scores as output. Recommending an $r$-ary transformation for $r$ features involves applying all $\left|\mathcal{T}_{r}\right|$ MLPs on $R_{\left[f_{1}, \ldots, f_{r}\right]}$. If the highest confidence score obtained from classifiers is above a given threshold, LFE recommends the corresponding transformation to be applied on feature $f$. Let $g_{k}\left(R_{\left[f_{1}, \ldots, f_{r}\right]}\right)$ be the confidence score of the MLP corresponding to transformation $T_{k}$, and $\gamma$ is the threshold for confidence scores which we determined empirically. LFE recommends the transformation $T_{c}$, for features $\left[f_{1}, \ldots, f_{r}\right]$, as follows:

$$
\begin{gathered}
c=\underset{k}{\arg \max } g_{k}\left(R_{\left[f_{1}, \ldots, f_{r}\right]}\right) \\
\text { recommend : } \begin{cases}T_{c}, & \text { if } g_{c}\left(R_{\left[f_{1}, \ldots, f_{r}\right]}\right)>\gamma \\
\text { none, } & \text { otherwise }\end{cases}
\end{gathered}
$$

In the following sections, we describe how numerical features are represented to be the input of transformation MLPs. Next, we explain the process of collecting past feature engineering knowledge as training samples to train MLPs.

\subsection{Feature-Class Representation}

Transformations are used to reveal and improve significant correlation or discriminative information between features and class labels. The more pronounced this correlation, the higher the chance that a model can achieve a better predictive performance. Each LFE classifier learns the patterns of feature-class distributions for which the corresponding transformation has been effective in improving feature-class correlation. LFE represents feature $f$ in a dataset with $k$ classes as follows:

$$
R_{f}=\left[Q_{f}^{(1)} ; Q_{f}^{(2)} ; \ldots ; Q_{f}^{(k)}\right]
$$

where $Q_{f}^{(i)}$ is a fixed-sized representation of values in $f$ that are associated with class $i$. We call this representation $Q u a n-$ tile Sketch Array. Next, we describe how feature values associated to class $i$ are translated into representation $Q_{f}^{(i)}$, which is meant to capture the distribution of feature values.

Neural networks have been successful in learning representations for image and speech data [Bengio et al., 2013]. Others have proposed solutions for estimating a Probability
Distribution Function (PDF) in an n-dimensional space [Garrido and Juste, 1998]. However, it is not clear how existing representation learning and PDF learning approaches may be applied in the context of raw numerical data. The main challenges is the high variability in the size and the range of feature values (e.g., from 10 to millions). In our setting, features are data points represented with various numbers of dimensions (number of distinct feature values). Hence, Random Projection [Bingham and Mannila, 2001] for dimensionality reduction is not applicable. Although Recurrent Neural Networks can deal with varying input size, we aim at determining a fixed-size representation that captures the correlation between features and target classes.

Previous approaches have used hand-crafted meta-features, including information-theoretic and statistical meta-features, to represent datasets [Michie et al., 1994; Kalousis, 2002; Feurer et al., 2015]. Such meta-features aim at modelling the distribution of values in datasets. Performing fixed-size sampling of feature values is another approach for representing distributions. Samples extracted from features and classes are required to reflect the distribution of values in both feature and target classes. While stratified sampling solves the issue for one feature, it becomes more complex for multiple features with high correlation [Skinner et al., 1994]. Feature hashing has been used to represent features of type "string" as feature vectors [Weinberger et al., 2009]. Although feature hashing can be generalized for numerical values, it is not straightforward to choose distance-preserving hash functions that map values within a small range to the same hash value. In Section 5, we empirically show how LFE transformation classifiers perform using each of representations described above.

Quantile Sketch Array (QSA) uses quantile data sketch [Wang et al., 2013] to represent feature values associated with a class label. QSA is a non-parametric representation that enables characterizing the approximate Probability Distribution Function of values. QSA is closely related to the familiar concept of histogram, where data is summarized into a small number of buckets. Naumann et al. used quantile representation for numerical features to perform feature classification [Naumann et al., 2002]. We apply the exact brute-force approach to compute quantile sketch for numerical values [Wang et al., 2013], described as follows.

Let $\mathcal{V}_{k}$ be the bag of values in feature $f$ that are for the training data points with the label $c_{k}$ and $Q_{f}^{(i)}$ is the quantile sketch of $\mathcal{V}_{k}$. First, we scale these values to a predefined range $[l b, u b]$. Generating $Q_{f}^{(i)}$ involves bucketing all values in $\mathcal{V}_{k}$ into a set of bins. Given a fixed number of bins, $r$, the range $[l b, u b]$ is partitioned into $r$ disjoint bins of uniform width $w=\frac{u b-l b}{r}$. Assume, the range $[l b, u b]$ is partitioned into bins $\left\{b_{0}, \ldots, b_{r-1}\right\}$, where the bin $b_{j}$ is a range $[l b+j * w, l b+(j+1) * w)$. Function $B\left(v_{l}\right)=b_{j}$ associates the value $v_{l}$ in $\mathcal{V}_{k}$ to the bin $b_{j}$. Function $P\left(b_{j}\right)$ returns the number of feature values, that are bucketed in $b_{j}$. Finally, $I\left(b_{j}\right)=\frac{P\left(b_{j}\right)}{\sum_{0 \leq m<r} P\left(b_{m}\right)}$ is the normalized value of $P\left(b_{j}\right)$ across all bins. 


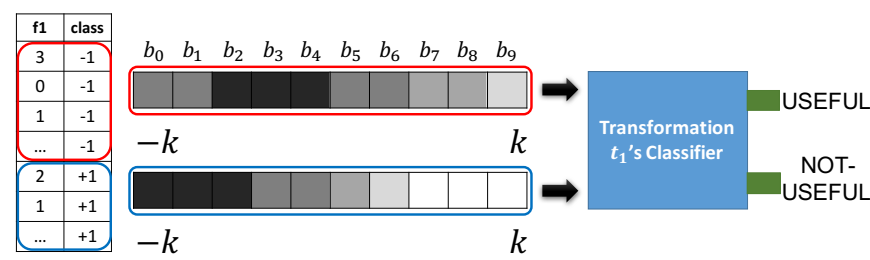

Figure 1: An example of feature representation using Quantile Sketch Array. The feature $f 1$ 's values are binned into 10 equi-width bins, separately for classes -1 and +1 .

To illustrate, Figure 1 shows an example of the representation of a feature. Note that this representation has certain behaviours. For example, for feature values that are perfectly separated in terms of the class label the representation ends up being very similar regardless of the scale of feature values. In this particular case there is also no need for any transformation since values are already separated. We decide QSA parameters (number of bins and scaling range) based on the performance of classifiers through empirical observations.

\subsection{Training}

To generate training samples for transformation MLP classifiers, LFE considers numerical features in classification datasets across various repositories. Each classifier is trained with the samples for which the corresponding transformation has been found useful as positive samples and all other samples as negative. In order to decide whether a transformation for a set of features leads to improvement, we evaluate a selected model, $\mathcal{L}$, on the original features and the target class as well as the constructed feature by itself and the target class. If the constructed feature shows performance improvement beyond a threshold, $\theta$, the input features together with their class labels are considered as a positive training sample for the transformation classifier.

A sample generated from feature $f$, in a dataset with $k$ classes, for transformation $t$ is translated into $R_{f}=$ $\left[Q_{f}^{(1)} ; \ldots ; Q_{f}^{(k)}\right]$. Assuming $b$ bins are used for each quantile data sketch, $R_{f}$ is a vector of size $k \times b$. Then, $R_{f}$ is fed into the MLP corresponding to $t$. Assume the corresponding MLP to the unary transformation $t$ has one hidden layer with $h$ units. The probability of $t$ being a useful transformation or not for feature $f$ in a dataset is computed as:

$$
\begin{aligned}
& {\left[p_{t \text { is useful }}(f), p_{t \text { is not useful }}(f)\right]=} \\
& \sigma_{2}\left(\mathbf{b}^{(\mathbf{2})}+\mathbf{W}^{(\mathbf{2})}\left(\sigma_{1}\left(\mathbf{b}^{(\mathbf{1})}+\mathbf{W}^{(\mathbf{1})}\left[Q_{f}^{(1)} ; \ldots ; Q_{f}^{(k)}\right]\right)\right)\right)
\end{aligned}
$$

where, $\mathbf{W}^{(\mathbf{1})}$ and $\mathbf{W}^{(\mathbf{2})}$ are weight matrices, $\mathbf{b}^{(\mathbf{1})}$ and $\mathbf{b}^{(\mathbf{1})}$ are bias vectors, and $\sigma_{1}$ and $\sigma_{2}$ are softmaxe and rectified linear unit (ReLU) functions, respectively [Nair and Hinton, 2010]. We use Stochastic Gradient Descent with minibatches to train transformation MLPs. In order to prevent overfitting, we apply regularization and drop-out [Srivastava et al., 2014]. The generated training samples are dependent on model type. In other words, while there may be overlaps in the suggested feature engineering paradigms across models, the optimal use of LFE for a specific model comes from using that same model while training LFE. In Section 5, we show that LFE is robust in terms of the choice of classification model.

\section{Experimental Results}

We evaluate three aspects of LFE: (1) the impact of using Quantile Sketch Array representation on the performance of transformation classifiers compared to other representations, (2) the capability of LFE in recommending useful transformations, (3) the benefit of using LFE to perform feature engineering compared to other alternatives, in prediction accuracy and time taken. We implemented LFE transformation classifiers and the meta-feature learner (auto-encoder) in TensorFlow. Transformation computation and model training were implemented using Scikit-learn. To showcase the capabilities of LFE, we considered the following ten unary and four binary transformations, respectively: log, square-root (both applied on the absolute of values), frequency (count of how often a value occurs), square, round, tanh, sigmoid, isotonic regression, zscore, normalization (mapping to $[-1,1]$ ), sum, subtraction, multiplication and division. In order to avoid data leakage, we apply transformations on train and test folds separately.

Without the loss of generality, we focus on binary classification for the purpose of these experiments. We collected 900 classification datasets from the OpenML and UCI repositories to train transformation classifiers. A subset of 50 datasets were reserved for testing and were not used for the purpose of training. In order to better utilize the datasets, we converted the multi-class problems into one-vs-all binary classification problems. Training samples were generated for Random Forest and Logistic Regression using 10-fold cross validation and the performance improvement threshold, $\theta$, of $1 \%$. Different improvement thresholds result in collecting variable number of training samples. Since the distribution of collected samples for transformations is not uniform, we decided on the threshold upon due empirical exploration. We generated approximately $84.5 \mathrm{~K}$ training samples for unary transformation and $122 \mathrm{~K}$ for binary transformations. Table 1 reports the statistics of positive training samples generated from all datasets. Frequency and multiplication are two transformations that incur performance improvement for the majority of features. All transformation classifiers are MLPs with one hidden layer. We tuned the number of hidden units to optimize the F-score for each classifier, and they vary from 400 to 500. The average time to train transformation classifiers offline was 6 hours.

\subsection{Feature Representation}

We evaluate the efficacy of Quantile Sketch Array (QSA) through the performance of LFE consisting of QSA compared to other representations, as listed in Table 2. These representations are listed in Table 2.

For hand-crafted meta-feature representation, we consider the following meta-features used in the literature: first 30 moments of feature values, median, standard deviation, min, $\max$, number of values and its log. For sampling representation, we obtain 250 stratified random samples per class. We also perform meta-feature learning by training an autoencoder on all features in our corpus. The input and output of the auto-encoder are the feature hashing representation [Weinberger et al., 2009] of features with 200 hash values per class. The auto-encoder consists of a two-layer en- 
Proceedings of the Twenty-Sixth International Joint Conference on Artificial Intelligence (IJCAI-17)

\begin{tabular}{llllllcccc|ccccc}
\hline Transformation & log & sqrt & square & freq & round & tanh & sigmoid & isotonic-reg & zscore & normalize & sum & subt & mult & div \\
\hline \#Positive Training Samples & 6710 & 6293 & 5488 & 73919 & 15855 & 70829 & 70529 & 624 & 664 & 31019 & 28492 & 36296 & 37086 & 19020 \\
\hline Classifier Performance & 0.95 & 0.96 & 0.97 & 0.77 & 0.92 & 0.92 & 0.80 & 0.98 & 0.97 & 0.91 & 0.98 & 0.99 & 0.97 & 0.98 \\
\hline
\end{tabular}

Table 1: Statistics of Training Samples and F1 Score of LFE Classifiers for 10-fold Cross Validation of Random Forest.

\begin{tabular}{lccc}
\hline $\begin{array}{l}\text { Hand-crafted } \\
\text { Meta-features }\end{array}$ & $\begin{array}{c}\text { Stratified } \\
\text { Sampling }\end{array}$ & $\begin{array}{c}\text { Meta-feature } \\
\text { Learning }\end{array}$ & $\begin{array}{c}\text { Quantile } \\
\text { Sketch Array }\end{array}$ \\
\hline 0.5558 & 0.5173 & 0.3256 & $\mathbf{0 . 9 1 2 9}$ \\
\hline
\end{tabular}

Table 2: F1 Score of Transformation Classifiers.

coder and a two-layer decoder, which are symmetric and each has a layer of 100 units connected to another layer of 75 units. For a hashed input feature, we consider the output of the encoder component of a trained auto-encoder as learned metafeatures of the feature. Finally, for Quantile Sketch Array, we consider scaling range of $[-10,10]$ and quantile data sketch size of 200 bins.

We use the same set of samples for training classifiers of different representations and tuned the MLPs to have their best possible configuration. In order to overcome the imbalance data classification problem, as shown in Table 1, we oversample the minority class samples to have balanced negative and positive samples during the training phase. The average 10-fold cross validation F1-score of a subset of classifiers is reported in Table 2 as a proxy of the benefit of representations. There is a clear distinction in terms of predictive performance and employed representation. The poorest results are obtained by learned meta-features, possibly since feature values belong to various ranges and hashing representation is not distance preserving, therefore, it is challenging for the auto-encoder to learn useful meta-features. Hand-crafted meta-features perform better than sampling and learned metafeatures, however, Quantile Sketch Array outperforms other representations by a significant margin $(35.7 \%)$.

\subsection{Transformation Classifier}

To evaluate the predictive power of each classifier, we trained classifiers corresponding to unary and binary transformations, using 10 -fold cross validation on all training samples. During the test phase, the corresponding transformation of each classifier is applied on one or a pair of feature(s) and the F1 score of the model based on the transformed feature only is evaluated. The F1-Score of classifiers using Random Forest and improvement threshold of 1\%, are shown in Table 1 . We considered $0 / 1$ loss evaluation. Table 1 demonstrates the high predictive capability of LFE transformation classifiers. As baseline, a random transformation recommender converged to F1 score of 0.50 for all transformation classifiers.

\subsection{Transformation Recommender}

To showcase the benefits of LFE in recommending useful features, we compare the predictive performance of test datasets augmented with features engineered by $L F E$ and features engineered by the following approaches.

- Random, which iterates for a given $r$ runs and in each run recommends a random or no transformation for feature(s). The performance of the dataset augmented with features constructed by randomly selected transformations is evalu- ated in each run and the transformations in the run with the highest predictive performance are recommended.

- Majority, which always recommends the single most effective transformation in the training samples (i.e. frequency for unary and multiplication for binary transformations).

- Brute-force, inspired by Feature Synthesis component of Data Science Machine [Kanter and Veeramachaneni, 2015], enumerates the whole feature space by applying all transformations to all features and performs feature selection on the augmented dataset.

- Model evaluation-based, which chooses the useful transformation for a feature by model evaluation on each constructed feature by each transformation. Having $t$ unary transformations, this approach performs $t$ model training runs for each feature.

To compare LFE with other feature engineering approaches, we consider 50 binary classification datasets. The detailed statistics of 23 of test datasets are shown in Table 3. Test datasets have a diverse range in number of features (2 to $10,936)$ and data points (57 to 140,707). All experimental evaluations are based on 10-fold cross validation on a Random Forest model. Since Quantile Sketch Array considers both feature values and class labels, in order to avoid data leakage problem, we do not consider the entire dataset when $L F E$ computes the recommendations. For experiments with unary transformations for each fold, we ask LFE to recommend at most one transformation for each feature in the train data fold. Next, recommended transformations are applied on the corresponding features of the test data fold and added as new features to the dataset. To only analyze the capability of feature engineering approaches, we do not perform any feature selection, except for brute-force approach to which feature selection is essential.

Table 3 compares the predictive performance and execution time of $L F E$ to other feature engineering approaches on test datasets. All columns in this table, except the last, report results when using unary transformations. Since 50\% of datasets time out for evaluation-based approach and $62 \%$ for brute-force approach, we only report the performance of LFE for binary transformations. All reported times in Table 3 include preparing data, recommending transformations, applying transformations and model training. Since the cost of training classifiers is one-time and offline, we do not consider it while comparing the runtime performance. LFE consistently outperforms all approaches on most datasets at a small cost of execution time. We argue that it is effective to spend certain effort offline in order to provide prompt answers at runtime. No feature engineering approach results in improvement of the performance of three datasets sonar and spambase and twitter-absolute. The evaluation-based approach on AP-omentum-lung, convex, gisette and higgs-boson timed out due to the excessive number of model training calls. Since the 
Proceedings of the Twenty-Sixth International Joint Conference on Artificial Intelligence (IJCAI-17)

\begin{tabular}{|c|c|c|c|c|c|c|c|c|c|}
\hline Dataset & $\begin{array}{c}\text { \#Numerical } \\
\text { Features }\end{array}$ & $\begin{array}{l}\text { \#Data } \\
\text { Points }\end{array}$ & $\begin{array}{c}\text { Base } \\
\text { Dataset }\end{array}$ & Majority & $\begin{array}{l}\text { Brute- } \\
\text { Force }\end{array}$ & $\begin{array}{l}\text { Random } \\
\text { (10 runs) }\end{array}$ & $\begin{array}{c}\begin{array}{c}\text { Evaluation } \\
\text { based }\end{array} \\
\end{array}$ & $\begin{array}{l}\text { unary } \\
\text { LFE }\end{array}$ & $\begin{array}{c}\text { binary } \\
\text { LFE }\end{array}$ \\
\hline AP-omentum-lung & 10936 & 203 & 0.883 & $\underline{0.915}$ & 0.925 & $\underline{0.908}$ & - & 0.929 & 0.904 \\
\hline AP-omentum-ovary & 10936 & 275 & 0.724 & $\overline{0.775}$ & $\overline{0.801}$ & $\overline{0.745}$ & $\underline{0.788}$ & 0.811 & $\overline{0.775}$ \\
\hline autos & 48 & 4562 & 0.946 & $\underline{0.95}$ & $\overline{0.944}$ & $\overline{0.929}$ & $\overline{0.954}$ & 0.96 & $\overline{0.949}$ \\
\hline balance-scale & 8 & 369 & 0.884 & $\overline{0.916}$ & $\underline{0.892}$ & 0.881 & $\overline{0.882}$ & 0.919 & $\overline{0.884}$ \\
\hline convex & 784 & 50000 & 0.82 & 0.5 & $\overline{0.913}$ & 0.5 & - & 0.819 & $\underline{0.821}$ \\
\hline credit-a & 6 & 690 & 0.753 & 0.647 & 0.521 & 0.643 & 0.748 & 0.771 & $\overline{0.771}$ \\
\hline dbworld-bodies & 2 & 100 & 0.93 & $\underline{0.939}$ & 0.927 & 0.909 & 0.921 & 0.961 & $\overline{0.923}$ \\
\hline diabetes & 8 & 768 & 0.745 & $\overline{0.694}$ & 0.737 & 0.719 & 0.731 & 0.762 & 0.749 \\
\hline fertility & 9 & 100 & 0.854 & $\underline{0.872}$ & $\underline{0.861}$ & 0.832 & 0.833 & 0.873 & $\overline{0.861}$ \\
\hline gisette & 5000 & 2100 & 0.941 & $\overline{0.601}$ & $\overline{0.741}$ & 0.855 & - & 0.942 & $\overline{0.933}$ \\
\hline hepatitis & 6 & 155 & 0.747 & 0.736 & $\underline{0.753}$ & 0.727 & 0.814 & $\underline{0.807}$ & $\underline{0.831}$ \\
\hline higgs-boson-subset & 28 & 50000 & 0.676 & 0.584 & $\overline{0.661}$ & 0.663 & - & $\overline{0.68}$ & $\overline{0.677}$ \\
\hline ionosphere & 34 & 351 & 0.931 & 0.918 & 0.912 & 0.907 & 0.913 & 0.932 & $\overline{0.925}$ \\
\hline labor & 8 & 57 & 0.856 & 0.827 & 0.855 & 0.806 & $\underline{0.862}$ & 0.896 & 0.896 \\
\hline lymph & 10936 & 138 & 0.673 & 0.664 & 0.534 & 0.666 & 0.727 & 0.757 & 0.719 \\
\hline madelon & 500 & 780 & 0.612 & 0.549 & 0.585 & 0.551 & $\overline{0.545}$ & 0.617 & $\overline{0.615}$ \\
\hline megawatt1 & 37 & 253 & 0.873 & $\underline{0.874}$ & $\underline{0.882}$ & 0.869 & $\underline{0.877}$ & 0.894 & $\overline{0.885}$ \\
\hline pima-indians-subset & 8 & 768 & 0.74 & 0.687 & $\overline{0.751}$ & 0.726 & 0.735 & $\underline{0.745}$ & 0.76 \\
\hline secom & 590 & 470 & 0.917 & 0.917 & 0.913 & 0.915 & 0.915 & 0.918 & 0.915 \\
\hline sonar & 60 & 208 & 0.808 & 0.763 & 0.468 & 0.462 & 0.806 & 0.801 & 0.783 \\
\hline spambase & 57 & 4601 & 0.948 & 0.737 & 0.39 & 0.413 & 0.948 & 0.947 & 0.947 \\
\hline spectf-heart & 43 & 80 & 0.941 & 0.955 & 0.881 & $\underline{0.942}$ & 0.955 & 0.955 & $\underline{0.956}$ \\
\hline twitter-absolute & 77 & 140707 & 0.964 & 0.866 & 0.946 & 0.958 & 0.963 & 0.964 & 0.964 \\
\hline \multirow{2}{*}{\multicolumn{2}{|c|}{$\begin{array}{l}\text { Feature Engineering and Model } \\
\text { Evaluation Time (seconds) }\end{array}$}} & geomean & 2.66 & 11.06 & 48.54 & 69.57 & 403.81 & 18.28 & 44.58 \\
\hline & & average & 19.23 & 1219.34 & 13723.51 & 2041.52 & 10508.75 & 97.90 & 188.17 \\
\hline
\end{tabular}

Table 3: Statistics of Datasets and F1 Score of LFE and Other Feature Engineering Approaches with 10-fold Cross Validation of Random Forest. The best performing approach is shown in bold for each dataset. The improving approaches are underlined.

evaluation-based approach requires model training for each combination of feature and transformation, the evaluationbased approach is only applicable to datasets with small number of features and data points.

To broaden the scope of experiments, we performed feature engineering using the same approaches and setting of Table 3 on 50 test datasets. Figure 2 shows the percentage of test datasets whose predictive performance improved by each feature engineering approach. For Random Forest, we observed that for $24 \%$ of test datasets, none of the considered approaches, including LFE, can improve classification performance. In all remaining datasets, LFE always improves the predictive performance and for $89 \%$ of such datasets LFE generates features that results in the highest classification performance. For the remaining $11 \%$ of datasets, the higher performance is achieved by other approaches at higher computational cost than LFE. To investigate the robustness of LFE with respect to the model, we performed the experiments for Logistic Regression and similar results were observed. While LFE is a one shot approach it already achieves an average improvement of more than $2 \%$ in F1 score and a maximal improvement of $13 \%$ across the 50 test data sets.

\section{Conclusion and Future Work}

In this paper, we present a novel framework called LFE to perform automated feature engineering by learning patterns between feature characteristics, class distributions, and useful transformations, from historical data. The cornerstone of our framework is a novel feature representation, called Quantile Sketch Array (QSA), that reduces any variable sized fea-

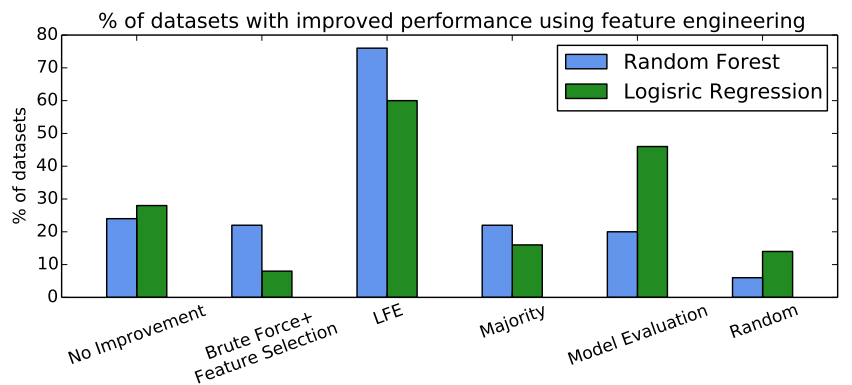

Figure 2: The percentage of datasets, from a sample of 50, for which a feature engineering approach results in performance improvement (measured by F1 score of 10 fold cross validation for Random Forest and Logistic Regression).

tures to a fixed size array, preserving its essential characteristics. QSA enables LFE to learn and predict the ability of a transform to improve the accuracy of a given dataset. Our empirical evaluation demonstrates the efficacy and efficiency of LFE in improving the predictive performance at low computational costs for a variety of classification problems. We plan to add more transformations, to design an iterative version of LFE, and combine it with exploration-based methods to achieve even more pronounced improvements. In addition, we aim to use the family of Recurrent Neural Networks to deal with both varying data set sizes and taking into account relationships across multiple features within a dataset to improve transformation recommendation. 


\section{References}

[Bengio et al., 2013] Yoshua Bengio, Aaron Courville, and Pascal Vincent. Representation learning: A review and new perspectives. IEEE TPAMI, 35(8), 2013.

[Bingham and Mannila, 2001] Ella Bingham and Heikki Mannila. Random projection in dimensionality reduction: Applications to image and text data. KDD, pages 245-250, 2001.

[Che et al., 2015] Zhengping Che, Sanjay Purushotham, Robinder Khemani, and Yan Liu. Distilling knowledge from deep networks with applications to healthcare domain. arXiv preprint arXiv:1512.03542, 2015.

[Dor and Reich, 2012] Ofer Dor and Yoram Reich. Strengthening learning algorithms by feature discovery. Information Sciences, 2012.

[Fan et al., 2010] Wei Fan, Erheng Zhong, Jing Peng, Olivier Verscheure, Kun Zhang, Jiangtao Ren, Rong Yan, and Qiang Yang. Generalized and heuristic-free feature construction for improved accuracy. SDM, 2010.

[Feurer et al., 2015] Matthias Feurer, Aaron Klein, Katharina Eggensperger, Jost Tobias Springenberg, Manuel Blum, and Frank Hutter. Efficient and robust automated machine learning. NIPS, 2015.

[Garrido and Juste, 1998] Lluis Garrido and Aurelio Juste. On the determination of probability density functions by using neural networks. Computer Physics Communications, 115(1):25 - 31, 1998.

[Greenwald and Khanna, 2001] Michael Greenwald and Sanjeev Khanna. Space-efficient online computation of quantile summaries. SIGMOD, pages 58-66, 2001.

[Kalousis, 2002] Alexandros Kalousis. Algorithm selection via meta-learning. $\mathrm{PhD}$ thesis, Universite de Geneve, 2002.

[Kanter and Veeramachaneni, 2015] James Max Kanter and Kalyan Veeramachaneni. Deep feature synthesis: Towards automating data science endeavors. DSAA, 2015.

[Katz et al., 2016] Gilad Katz, Eui Chul Richard Shin, and Dawn Song. Explorekit: Automatic feature generation and selection. ICDM, pages 979-984, 2016.

[Khurana et al., 2016] Udayan Khurana, Deepak Turaga, Horst Samulowitz, and Srinivasan Parthasarathy. Cognito: Automated Feature Engineering for Supervised Learning. ICDM, 2016.

[Lichman, 2013] M. Lichman. UCI machine learning repository, 2013.

[Markovitch and Rosenstein, 2002] Shaul Markovitch and Dan Rosenstein. Feature generation using general constructor functions. Machine Learning, 2002.

[Michie et al., 1994] Donald Michie, D. J. Spiegelhalter, C. C. Taylor, and John Campbell, editors. Machine Learning, Neural and Statistical Classification. 1994.

[Nair and Hinton, 2010] Vinod Nair and Geoffrey E. Hinton. Rectified linear units improve restricted boltzmann machines. ICML, pages 807-814, 2010.
[Naumann et al., 2002] F. Naumann, C.-T. Ho, X. Tian, L. Haas, and $\mathrm{N}$ Megiddo. Attribute classification using feature analysis. ICDE, page 271, 2002.

[Rifkin and Klautau, 2004] Ryan Rifkin and Aldebaro Klautau. In defense of one-vs-all classification. JMLR, 5:101141, 2004.

[Shawe-Taylor and Cristianini, 2004] John Shawe-Taylor and Nello Cristianini. Kernel methods for pattern analysis. Cambridge university press, 2004.

[Skinner et al., 1994] C.J. Skinner, D.J. Holmes, and D. Holt. Multiple frame sampling for multivariate stratification. International Statistical Review, 62(3), 1994.

[Srivastava et al., 2014] Nitish Srivastava, Geoffrey Hinton, Alex Krizhevsky, Ilya Sutskever, and Ruslan Salakhutdinov. Dropout: A simple way to prevent neural networks from overfitting. Journal of Machine Learning, 15(1):1929-1958, 2014.

[Storcheus et al., 2015] Dmitry Storcheus, Afshin Rostamizadeh, and Sanjiv Kumar. A survey of modern questions and challenges in feature extraction. Proceedings of The 1st International Workshop on Feature Extraction, NIPS, 2015.

[Vanschoren et al., 2014] Joaquin Vanschoren, Jan N. van Rijn, Bernd Bischl, and Luis Torgo. Openml: Networked science in machine learning. SIGKDD Explor. Newsl., 15(2):49-60, June 2014.

[Wang et al., 2013] Lu Wang, Ge Luo, Ke Yi, and Graham Cormode. Quantiles over data streams: An experimental study. SIGMOD, pages 737-748, 2013.

[Weinberger et al., 2009] Kilian Weinberger, Anirban Dasgupta, John Langford, Alex Smola, and Josh Attenberg. Feature hashing for large scale multitask learning. ICML, 2009. 